\title{
Trách nhiệm xã hội của doanh nghiệp, tài sản thương hiệu và kết quả kinh doanh dựa trên cảm nhận của nhân viên công ty du lịch trong bối cảnh hội nhập TPP
}

\section{Correlation among corporate social responsibility, brand equity and firm performance based on feeling of staff in travel companies in TPP integration}

\author{
Hoàng Anh Viện ${ }^{1 *}$ \\ ${ }^{1}$ Trường Đại học Công nghiệp Thành phố Hồ Chí Minh - Phân hiệu Quảng Ngãi, Việt Nam \\ *Tác giả liên hệ, Email: viendhcn@gmail.com
}

THÔNG TIN

DOI: $10.46223 /$ HCMCOUJS.

econ.vi.13.3.1512.2018

Ngày nhận: 01/11/2016

Ngày nhận lại: 10/07/2018

Duyệt đăng: 10/07/2018

Tù khóa:

doanh nghiệp du lịch, kết quả

kinh doanh, tài sản thương

hiệu, trách nhiệm xã hội
TÓM TĂT

Nghiên cứu này nhằm xác định mối quan hệ giữa nhận thức CSR của nhân viên đối với tài sản thương hiệu và kết quả kinh doanh trong ngành du lịch. Số liệu được thu thập từ 336 nhân viên của các doanh nghiệp du lịch thông qua bảng câu hỏi được thiết kế sẵn. Các phương pháp thống kê so sánh, mô tả, tổng hợp; kiểm định Cronbach's Alpha, phân tích nhân tố khám phá EFA, phân tích nhân tố khẳng định $\mathrm{CFA}$ và phân tích mô hình cấu trúc tuyến tính SEM được dùng để giải thích dữ liệu. Kết quả cho thấy cả 4 yếu tố: cảm nhận của nhân viên về trách nhiệm kinh tế, trách nhiệm đạo đức, trách nhiệm pháp lý và trách nhiệm từ thiện đều có tác động tích cực đến tài sản thương hiệu và kết quả kinh doanh. Ngoài ra, tài sản thương hiệu có tác động tích cực đến kết quả kinh doanh. Nghiên cứu cũng đưa ra một số gợi ý chính sách cho các nhà quản lý công ty du lịch trong việc nâng cao các hoạt động trách nhiệm xã hội của mình.

\section{ABSTRACT}

The aim of this paper is to identify the relationship between staff Corporate Social Responsibility (CSR) awareness of brand equity and firm performance in the tourism sector using a welldesigned questionnaire to collect data from 336 staff working in tourism enterprises. The research uses various models of Cronbach's Alpha coefficient, Exploratory Factor Analysis (EFA), Confirmatory Factor Analysis (CFA), and Structural Equation Analysis (SEM) to analyze the data. Research results show that all four factors of staff feelings about economic responsibilities, ethical responsibilities, legal responsibilities and philanthropic responsibilities have a positive impact on brand 
Keywords:

tourism enterprises, firm performance, brand equity, CSR equity and firm performance. Besides, brand equity has a positive impact on firm performance. The study also suggests some implications for tourism managers to enhance CSR activities in their business.

\section{1. Đặt vấn đề}

Khi tham gia TPP, lĩnh vực du lịch của Việt Nam sẽ phát triển đáng kể do sự gia tăng của dòng khách trong khối đến tìm kiếm cơ hội kinh doanh và đầu tư. Việc nới lỏng điều kiện tự do đầu tư và di chuyển lao động giữa 12 nước sẽ giúp gia tăng nhu cầu đi lại tìm kiếm cơ hội đầu tư, việc làm và kêt hợp du lịch; gia tăng dòng du khách quốc tế và tăng cường mật độ, quy mô các loại hình du lịch - kinh doanh, du lịch - hội họp (MICE) ngay trong nội khối TPP mà Việt Nam là một thành viên. Du khách quốc tế có cơ hội làm thủ tục thuận lợi, dịch chuyển nhanh và rẻ hơn bởi có những cam kết nhập cảnh tạm thời của khách kinh doanh trong khuôn khổ TPP... (Dinh, 2016).

Điều đáng nói là $5 / 12$ nước thành viên của TPP gồm Hoa Kỳ, Việt Nam, Malaysia, Australia và Mexico đều nằm trong danh sách các nước đa dạng sinh thái hàng đầu thế giới, với việc siết chặt các yêu cầu về vệ sinh và môi trường sống, chống buôn bán động vật hoang dã, ... Do đó môi trường sống và cơ sở hạ tầng du lịch thiên nhiên sẽ được cải thiện hơn khi thực hiện cam kết trong TPP. Điều này thúc đẩy du lịch phát triển bền vững và có trách nhiệm.

Tại Việt Nam, vấn đề trách nhiệm xã hội của doanh nghiệp đã được một số bộ, ngành quan tâm, chú ý khá sớm. Bằng chứng là, từ năm 2005, Phòng Thương mại và Công nghiệp Việt Nam, Bộ Lao động Thương binh và Xã hội, Bộ Công thương cùng với các hiệp hội Da giày, Dệt may trao giải thưởng "Trách nhiệm xã hội của doanh nghiệp hướng tới sự phát triển bền vững" nhằm tôn vinh các doanh nghiệp thực hiện tốt trách nhiệm xã hội của doanh nghiệp trong bối cảnh hội nhập. Nhiều doanh nghiệp lớn ở Việt Nam như Samsung Electronics Việt Nam, Unilever Việt Nam, Vinamilk... xem CSR là một triết lý kinh doanh cơ bản và song hành với chiến lược phát triển góp phần vào sự thành công bền vững, giúp doanh nghiệp thực hiện được tầm nhìn và sứ mệnh. Việc thực hành $\mathrm{CSR}$ của các doanh nghiệp và người sản xuất không những góp phần đảm bảo an sinh xã hội, an toàn lao động, đảm bảo môi trường bền vững mà còn là yếu tố sống còn để xây dựng và phát triển tài sản thương hiệu và nâng cao kết quả kinh doanh của doanh nghiệp.

Các nghiên cứu trước đây cho rằng, CSR dẫn đến hành vi đạo đức của nhân viên, từ đó tăng cường hiệu quả tổ chức (Laczniak \& Murphy, 1991; Preston \& O'Bannon, 1997; Sims \& Kroeck, 1994). Thực hiện đầy đủ CSR sẽ cải thiện hình ảnh doanh nghiệp và tăng cường ảnh hưởng của chiến thuật marketing, do đó ảnh hưởng tích cực đến hiệu suất công ty (Maignan, Ferrell, \& Frell, 2005). Torres, Bijmolt, Tribo, và Verhoef (2012) nhận thấy rằng, thực hiện CSR đối với tất cả các bên liên quan ảnh hưởng tích cực đến tài sản thương hiệu.

Lai, Chiu, Yang, và Pai (2010) nghiên cứu ảnh hưởng của CSR lên hiệu suất thương hiệu trong thị trường công nghiệp (B2B) với phương pháp nghiên cứu định tính thông qua bảng câu hỏi. Kết quả cho thấy rằng $\mathrm{CSR}$ ảnh hưởng tích cực đến tài sản thương hiệu công nghiệp và hiệu suất thương hiệu. 
Ngành Du lịch đang chủ trương thực hiện chính sách phát triển bền vững, trong đó coi phát triển du lịch có trách nhiệm là con đường dẫn đến sự thành công. Doanh nghiệp du lịch đóng một vai trò quan trọng trong việc xây dựng, quảng bá và cung cấp sản phẩm du lịch có trách nhiệm đến với người tiêu dùng. Nâng cao nhận thức của nhân viên về CSR thông qua đó góp phần tăng cường tài sản thương hiệu và nâng cao hiệu quả sản xuất kinh doanh của doanh nghiệp sẽ hỗ trợ họ thực hiện các vai trò quan trọng như là "nhà cung cấp" du lịch có trách nhiệm và đóng góp quan trọng vào sự phát triển chung, nâng cao chất lượng sản phẩm du lịch cũng như xây dựng hình ảnh Việt Nam như là điểm đến du lịch có trách nhiệm. Chính vì lý do đó bài viết này tập trung nghiên cứu mối quan hệ giữa nhận thức CSR của nhân viên, tài sản thương hiệu và kết quả kinh doanh - Trường hợp các doanh nghiệp du lịch tại Thành phố Hồ Chí Minh. Mục tiêu trọng tâm của bài báo: (1) Nghiên cứu tác động của CSR đến tài sản thương hiệu; (2) Nghiên cứu tác động của CSR đến kết quả kinh doanh; (3) Nghiên cứu tác động của tài sản thương hiệu đến kết quả kinh doanh.

\section{Co' sở lý thuyết và mô hình nghiên cứu}

\subsection{Trách nhiệm xã hội của doanh nghiệp (CSR)}

Có nhiều định nghĩa CSR đã được đưa ra, theo Bowen (1953), CSR là nghĩa vụ của thương nhân theo đuổi các chính sách để đưa ra quyết định hoặc những hành động cần thiết về các mục tiêu và giá trị cho xã hội. McGuire (1963) cho rằng một công ty không chỉ có nghĩa vụ về mặt kinh tế và chấp hành luật pháp, mà còn phải có những trách nhiệm nhất định khác đối với xã hội. Theo ý tưởng này, Carroll $(1979,1991)$ đã khái quát hóa các trách nhiệm xã hội thành bốn nhóm: trách nhiệm kinh tế, trách nhiệm luật pháp, trách nhiệm đạo đức và trách nhiệm từ thiện. Quan điểm của Carroll về CSR thể hiện cụ thể trong Hình 1.

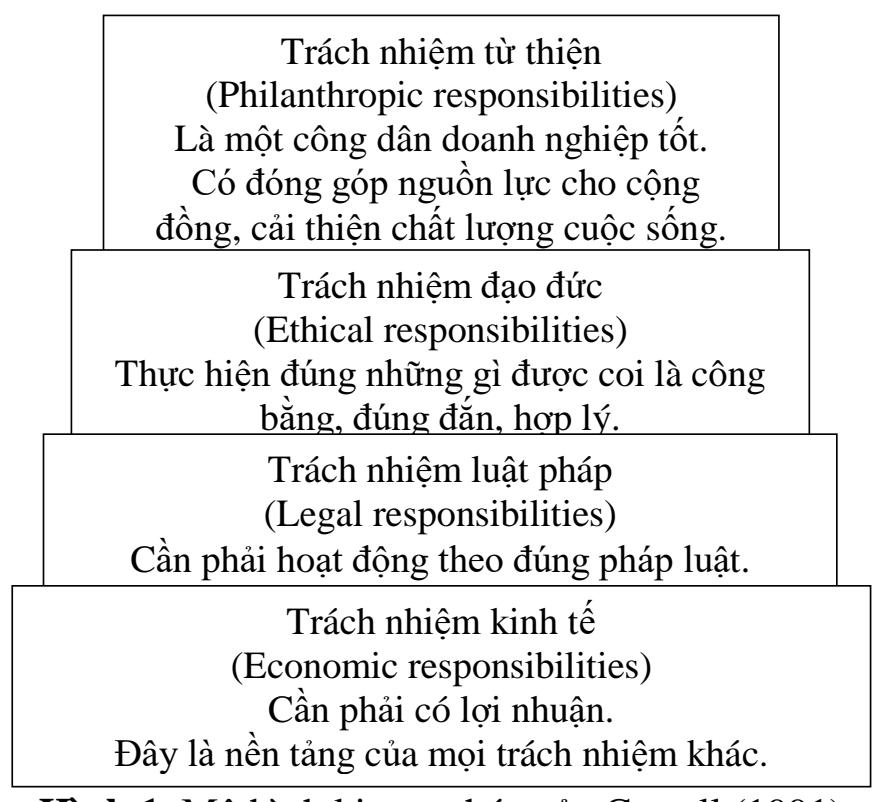

Hình 1. Mô hình kim tự tháp của Carroll (1991)

Trách nhiệm kinh tế tức là doanh nghiệp có nghĩa vụ tạo ra lợi nhuận; cung cấp việc làm; và sản xuất ra sản phẩm/dịch vụ mà khách hàng cần. Trách nhiệm luật pháp là doanh nghiệp phải có nghĩa vụ tuân theo các luật lệ, luật pháp của địa phương, của đất nước và cả luật 
quốc tế trong quá trình hoạt động. Trách nhiệm đạo đức là doanh nghiệp phải đáp ứng các chuẩn mực, kỳ vọng khác của xã hội, những điều không được ghi trong luật; cụ thể hơn là trách nhiệm đáp ứng được các chuẩn mực, kỳ vọng của các bên liên quan, gồm có khách hàng, nhân viên, cổ đông và cộng đồng. Trách nhiệm từ thiện tức là doanh nghiệp phải đáp ứng được các kỳ vọng từ xã hội, doanh nghiệp cũng nên giống như là những công dân tốt; thí dụ như đóng góp về nguồn lực tài chính, giáo dục, hoạt động cộng đồng.

Mô hình kim tự tháp của Carroll (1991) là quan điểm được nhiều học giả chấp nhận và nhà nghiên cứu ứng dụng (Hoang \& Huynh, 2015). Nghiên cứu này dựa trên phương pháp luận của mô hình kim tự tháp của Carroll (1991) và được đặt trong bối cảnh ngành du lịch Việt Nam.

\subsection{CSR và các bên liên quan}

Các bên liên quan là các cá nhân và các nhóm người có thể ảnh hưởng đến và có thể hành vi của họ bị ảnh hưởng bởi hoạt động của Công ty (Freeman, 1984). Là thành viên của tổ chức, nhân viên có liên quan, đóng góp, và phản ứng với đầu tư của nó trong thực hành trách nhiệm xã hội (Rupp, Gananpathy, Aguilera, \& Williams, 2006). Nhân viên không chỉ mong đợi công ty cư xử một cách có trách nhiệm xã hội, mà họ còn là tác nhân quan trọng của CSR (Peterson, 2004; Rupp et al., 2006). Vì vậy, cuối cùng việc thực thi các chiến lược CSR một cách thường xuyên là trách nhiệm của nhân viên. Thành tựu của các kế hoạch CSR sẽ phụ thuộc phần lớn vào sự tự nguyện của họ hợp tác và tuân thủ chiến lược CSR (Collier \& Esteban, 2007). Bên cạnh đó tầm quan trọng của họ như là tác nhân của CSR, nhân viên cũng đóng vai trò khác với sự gia tăng tiềm năng về quan tâm và nâng cao nhận thức của họ về thực tiễn thực hiện trách nhiệm xã hội của Công ty.

\subsection{Tài sản thuơng hiệu công nghiệp}

Khái niệm về tài sản thương hiệu đã được phát triển trong thị trường $\mathrm{B} 2 \mathrm{C}$ và được chấp nhận rằng, tài sản thương hiệu và quản trị thương hiệu là rất quan trọng cho sự thành công của các công ty $\mathrm{B} 2 \mathrm{C}$ (Jing, Yanxin, Rizwan, \& Mingfei, 2015). Nhiều tác giả cho rằng khái niệm xây dựng thương hiệu có khả năng áp dụng trong cả thị trường B2B và $\mathrm{B} 2 \mathrm{C}$ (Davis, Golicic, \& Marquardt, 2008). Nghiên cứu gần đây chỉ ra rằng tài sản thương hiệu có tầm quan trọng cao trong việc tạo vị thế cạnh tranh của các công ty B2B và hiệu suất (Backhaus, Steiner, \& Lügger, 2011; Kim \& Hyun, 2011; Kotler \& Pfoertsch, 2007).

Các nghiên cứu trước đây về xây dựng thương hiệu B2B đã chủ yếu tập trung vào việc xác định sự khác biệt giữa xây dựng thương hiệu trong người tiêu dùng so với các bối cảnh công nghiệp (e.g., Brown, Bellenger, \& Johnston, 2007). Gần đây các nhà nghiên cứu bắt đầu nghiên cứu khám phá các yếu tố tiền đề của tài sản thương hiệu công nghiệp, chẳng hạn như: giá trị so với tiền, hiệu suất phân phối, xúc tiến và nhân sự; (Van Riel, de Mortanges, \& Streukens, 2005) hình ảnh đoàn thể, chất lượng sản phẩm và dịch vụ, giá cả và chi phí (Cretu \& Brodie, 2007); chất lượng dịch vụ, đáp ứng, và trao quyền (Roberts \& Merrilees, 2007); chất lượng sản phẩm, chất lượng dịch vụ, giá cả, sự khác biệt, lời hứa, và sự tin tưởng và tín nhiệm (Jensen \& Klastrup, 2008); thẩm quyền nhà cung cấp và giá trị mua của người mua (Han \& Sung, 2008); kinh nghiệm khách hàng (Biedenbach \& Marell, 2010); Trách nhiệm xã hội và uy tín của công ty (Lai et al., 2010),... 


\subsection{Kết quả kinh doanh}

Đa số nhà nghiên cứu cho rằng kết quả kinh doanh của doanh nghiệp là mức độ đạt được mục tiêu của doanh nghiệp (Buzzell \& Gale, 1987; Cyert \& March, 1992). Kết quả kinh doanh thể hiện bằng lợi nhuận, tăng trưởng thị phần, doanh thu và các mục tiêu chiến lược của doanh nghiệp. Kết quả kinh doanh được đánh giá dựa trên mức độ đạt được các mục tiêu đó (Hult, Hurley, \& Knight, 2004).

\subsection{CSR và tài sản thương hiệu}

Smith và Higgins (2000) cho rằng: "Người quản lý thương hiệu sử dụng mối quan tâm của người tiêu dùng đối với trách nhiệm kinh doanh như là một phương tiện để đảm bảo lợi thế cạnh tranh". Brickley, Smith, và Zimmerman (2002) cũng thừa nhận rằng uy tín của công ty đối với hành vi trách nhiệm xã hội là một bộ phận quan trọng của tài sản thương hiệu doanh nghiệp. Maignan và Ferrell (2004) cho rằng CSR sẽ giúp công ty tăng cường lợi thế tiếp thị và củng cố bản sắc doanh nghiệp với các bên liên quan. Jones (2005) đề xuất rằng giá trị thương hiệu xuất phát từ các tương tác được tạo ra giữa thương hiệu và các bên liên quan của nó. Doanh nghiệp càng thực hiện được kỳ vọng của các bên liên quan, tài sản thương hiệu càng có giá trị hơn.

CSR ảnh hưởng tích cực đến giá trị thương hiệu công nghiệp (Lai et al., 2010). Demetriou, Mouyi, và Spanoudis (2010) lập luận rằng nếu một công ty tham gia vào các sáng kiến CSR, người tiêu dùng có cảm giác tốt hơn đối với các nhãn hiệu của doanh nghiệp. Thực hiện đầy đủ trách nhiệm xã hội có thể có một tác động tích cực về hình ảnh công ty của công ty (Huang \& Ho, 2012).

Khi nhân viên hiểu được doanh nghiệp họ đang tích cực tham gia các hoạt động CSR và điều này làm cho họ tự hào khi là một phần của doanh nghiệp đó (You et al., 2013). Khi đó, họ sẽ hỗ trợ tích cực hơn cho các hoạt động CSR và qua đó góp phần gia tăng tài sản thương hiệu cho doanh nghiệp. Theo logic này, nghiên cứu này suy luận rằng những nhận thức tích cực của nhân viên về việc thực hiện CSR của một công ty công nghiệp có thể làm tăng tài sản thương hiệu của công ty.

H1: Nhận thức về trách nhiệm kinh tế của nhân viên tác động thuận chiều với tài sản thuoong hiệu

H2: Nhận thức về trách nhiệm luật pháp của nhân viên tác động thuận chiều với tài sản thuoong hiệu

H3: Nhận thức về trách nhiệm đạo đức của nhân viên tác động thuận chiều với tài sản thuoong hiệu

H4: Nhận thức về trách nhiệm tù̀ thiện của nhân viên tác động thuận chiều với tài sản thương hiệu

\subsection{CSR và kết quả kinh doanh}

Laczniak và Murphy (1991) cho rằng khi công ty cam kết phát triển văn hóa về đạo đức kinh doanh sẽ tránh phát sinh các tổn thất cá nhân và tổ chức, và các chi phí xã hội, do đó dẫn đến một kết quả kinh doanh tốt hơn. Verschoor (1998) xem xét các dữ liệu tài chính của 
500 công ty và kết luận rằng CSR có một mối quan hệ nhân quả với kết quả kinh doanh. Preston và O'Bannon (1997), lập luận rằng có một mối quan hệ tích cực giữa CSR và hoạt động tài chính. Brammer và Millington (2008) chỉ ra rằng thực hiện CSR cao có thể giúp nâng cao lợi nhuận của một công ty thông qua việc giảm chi phí hoạt động và tăng doanh thu. Khi doanh nghiệp thực hiện CSR sẽ thể hiện họ là một nhà đầu tư có trách nhiệm, qua đó góp phần nâng cao kết quả kinh doanh (Chu \& Yang, 2009; Griffin \& Mahon, 1997; Peters \& Mullen, 2009; Y. G. Wang, Hsu, \& Chang, 2012).

Khi nhân viên hiểu được doanh nghiệp họ đang tích cực tham gia các hoạt động CSR và điều này làm cho họ tự hào khi là một phần của doanh nghiệp đó (You et al., 2013).

Khi đó, họ sẽ hỗ trợ tích cực hơn cho các hoạt động CSR và qua đó góp phần nâng cao hiệu quả sản xuất kinh doanh cho doanh nghiệp. Theo logic này, nghiên cứu này suy luận rằng những nhận thức tích cực của nhân viên về việc thực hiện CSR của một công ty công nghiệp có thể làm tăng kết quả kinh doanh của công ty.

H5: Nhận thức về trách nhiệm kinh tế của nhân viên tác động thuận chiều với kết quả kinh doanh

H6: Nhận thức về trách nhiệm kinh tế của nhân viên tác động thuận chiều với kết quả kinh doanh

H7: Nhận thức về trách nhiệm kinh tế của nhân viên tác động thuận chiều với kết quả kinh doanh

H8: Nhận thức về trách nhiệm kinh tế của nhân viên tác động thuận chiều với kết quả kinh doanh

\subsection{Tài sản thương hiệu và kết quả kinh doanh}

Mặc dù tài sản thương hiệu không phải là yếu tố duy nhất tác động đến kết quả kinh doanh, nhưng nó vẫn có tầm quan trọng rất lớn, bởi vì năng lực tiếp thị động, kết quả từ các tương tác của các bên liên quan được thể hiện trong tài sản thương hiệu, có thể cải thiện kết quả kinh doanh (Morgan, Slotegraaf, \& Vorhies, 2009; Vorhies, Orr, \& Bush, 2011). Một công ty có khả năng hợp tác thành công với các bên liên quan và tích hợp thông minh với các bên liên quan có nhiều khả năng xác định và đáp ứng các cơ hội thông qua các sản phẩm mới, dịch vụ và quy trình, do đó có thể tăng doanh thu (Ayuso, Rodríguez, \& Ricart, 2006; Dangelico, Pontrandolfo, \& Pujari, 2013; Weerawardena, O'Cass, \& Julian, 2006).

H9: Tài sản thuoong hiệu tác động thuận chiều tới kết quả kinh doanh

Chín giả thuyết trên được thể hiện trong mô hình nghiên cứu như Hình 2. 


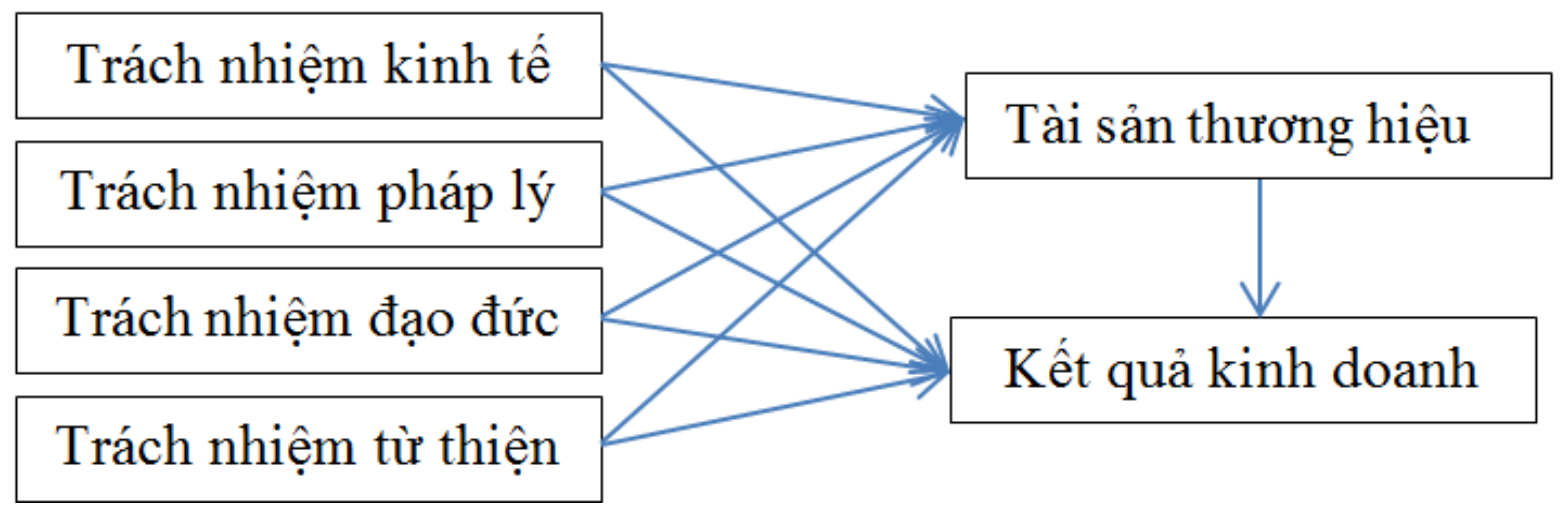

Hình 2. Mô hình nghiên cứu

\section{3. Đối tượng và phương pháp nghiên cứu}

\section{1. Đối tự̣ng nghiên cúu}

Đối tượng trong nghiên cứu này là mối quan hệ giữa trách nhiệm xã hội, tài sản thương hiệu và kết quả kinh doanh của doanh nghiệp Việt Nam.

Đối tượng khảo sát là các doanh nghiệp và cơ sở kinh doanh dịch vụ du lịch trên địa bàn Thành phố Hồ Chí Minh. Đối tượng được phỏng vấn là nhân viên của các đơn vị này.

\subsection{Phương pháp nghiên cúu}

Thang đo cảm nhận của nhân viên về thực hiện CSR của công ty, tác giả sử dụng thang đo nguyên gốc từ nghiên cứu trước của nhóm tác giả Hoang và Huynh (2015) và đã hiệu chỉnh sau nghiên cứu sơ bộ. Có 1 biến quan sát TNTT2 được điều chỉnh từ "Quan tâm hỗ trợ phát triển cộng đồng địa phương" thành "Quan tâm hỗ trợ bảo vệ môi trường điểm đến" cho phù hợp với điều kiện doanh nghiệp du lịch Thành phố Hồ Chí Minh.

Ngoài ra, đối với hoạt động du lịch và khách sạn thì khả năng tổ chức các liên minh, liên kết đóng một vai trò quan trọng trong chiến lược cạnh tranh (Heath, 2000; Hwang \& Chang, 2003; Pine \& Phillips, 2005; Preble, Reichel, \& Hoffman, 2000; Shaw \& Williams, 1990) do đó tác giả sử dụng thang đo tài sản thương hiệu cho thị trường B2B của các tác giả (Baumgarth \& Binckebanck, 2011; Baumgarth \& Schmidt, 2010; Davis et al., 2008; Han \& Sung, 2008). Thang đo kết quả kinh doanh dựa theo Wu và Cavusgil (2006) và Keh, Nguyen, và Hwei (2007).

Bài viết sử dụng phương pháp nghiên cứu định lượng. Thang đo và mô hình nghiên cứu được kiểm định bằng các phương pháp phân tích định lượng trên các phần mềm SPSS 20.0 và Amos 20.0. Tác giả thực hiện phỏng vấn trực tiếp nhân viên các doanh nghiệp và cơ sở kinh doanh dịch vụ du lịch trên địa bàn Thành phố Hồ Chí Minh bằng bảng câu hỏi. Bảng câu hỏi được gửi trực tiếp đến trước và hẹn ngày quay lại nhận. Thời gian khảo sát từ tháng 6 đến tháng 8 năm 2016. Số lượng bảng câu hỏi phát ra là 390, thu về với số mẫu hợp lệ là 336. 


\section{Kết quả nghiên cứu và thảo luận}

\section{1. Đặc điểm mẫu nghiên cúu}

Tổng số bảng câu hỏi phát ra là 390, thu về với số mẫu hợp lệ là 336 tương ứng với tỷ lệ $86,15 \%$ được sử dụng làm dữ liệu nghiên cứu. Trong số 336 , phân bố theo thời gian làm việc, loại hình và quy mô doanh nghiệp như Bảng 1 .

\section{Bảng 1}

Đặc điểm mẫu nghiên cứu

\begin{tabular}{|l|c|c|}
\hline \multicolumn{1}{|c|}{ Đặc điểm } & Tần số & Tỷ lệ (\%) \\
\hline Thò̀i gian làm việc: & & \\
- 1 đến 3 năm & 139 & 41.4 \\
- Trên 3 năm đến 5 năm & 123 & 36.6 \\
- Trên 5 năm đến 10 năm & 50 & 14.9 \\
- Trên 10 năm & 24 & 7.1 \\
\hline Loại hình doanh nghiệp: & & \\
- Công ty cổ phần & 25 & 7.4 \\
- Công ty TNHH & 174 & 51.8 \\
- Doanh nghiệp tư nhân & 76 & 22.6 \\
- Cơ sở kinh doanh & 61 & 18.2 \\
\hline Quy mô doanh nghiệp: & & \\
- 1 đến 50 nhân viên & 218 & 64.9 \\
- Trên 50 đến 100 nhân viên & 68 & 20.2 \\
- Trên 100 đến 200 nhân viên & 32 & 9.5 \\
- Trên 200 nhân viên & 18 & 5.4 \\
\hline Tổng & 336 & $100 \%$ \\
\hline
\end{tabular}

Nguồn: Kết quả phân tích dữ liệu của nhóm nghiên cứu

\subsection{Kiểm định thang đo bằng hệ số tin cậy và phân tích nhân tố khám phá (EFA)}

Kết quả phân tích Cronbach's Alpha lần 1 của các thang đo có các biến quan sát TNDD3, TNTT3, TNTT5, TNKT4 bị loại vì hệ số tương quan biến tổng không đạt yêu cầu. Kết quả phân tích lần 2 các thang đo có hệ số Cronbach's Alpha đạt yêu cầu $>0,6$ và các hệ số tương quan biến tổng $>0,3$. Vì vậy, các thang đo trong mô hình đạt tiêu chuẩn, đảm bảo độ tin cậy và được đưa vào phân tích nhân tố khám phá.

\section{Bảng 2}

Kết quả phân tích hệ số tin cậy Cronbach's Alpha

\begin{tabular}{|l|c|c|c|}
\hline \multicolumn{1}{|c|}{ Khái niệm } & Số biến & Cronbach's alpha & Hệ số tải \\
\hline Trách nhiệm kinh tế & 4 & 0.831 & $0.594-0.731$ \\
Trách nhiệm pháp lý & 5 & 0.879 & $0.676-0.772$ \\
\hline
\end{tabular}




\begin{tabular}{|l|c|c|c|}
\hline \multicolumn{1}{|c|}{ Khái niệm } & Số biến & Cronbach's alpha & Hệ số tải \\
\hline Trách nhiệm đạo đức & 4 & 0.888 & $0.728-0.808$ \\
Trách nhiệm từ thiện & 4 & 0.852 & $0.637-0.780$ \\
Tài sản thương hiệu & 5 & 0.890 & $0.697-0.753$ \\
Kết quả kinh doanh & 5 & 0.925 & $0.754-0.863$ \\
\hline
\end{tabular}

Nguồn: Kết quả xử lý từ dữ liệu điều tra

Kết quả phân tích nhân tố cho các thang đo thành phần của trách nhiệm xã hội, thang đo tài sản thương hiệu và kết quả kinh doanh có hệ số $\mathrm{KMO}>0,5 ; \mathrm{Sig} .=0,000<0,05$, tổng phương sai trích lớn $50 \%$. Như vậy kết quả phân tích nhân tố cho các thang đo đảm bảo yêu cầu, kết quả cụ thể ở Bảng 3 .

\section{Bảng 3}

Kết quả phân tích nhân tố khám phá EFA

\begin{tabular}{|l|c|l|c|c|}
\hline Khái niệm & KMO & Sig & Phương sai trích & Hệ số loading \\
\hline 1. Các biến độc lập về TNXH & 0.844 & .000 & $69.904 \%$ & \\
- Trách nhiệm kinh tế & & & & $0.753-0.849$ \\
- Trách nhiệm pháp lý & & & & $0.761-0.853$ \\
- Trách nhiệm đạo đức & & & & $0.820-0.880$ \\
- Trách nhiệm từ thiện & & & & $0.768-0.881$ \\
2. Tài sản thương hiệu & 0.867 & .000 & $69.765 \%$ & $0.809-0.851$ \\
3. Kết quả kinh doanh & 0.898 & .000 & $77.175 \%$ & $0.840-0.917$ \\
\hline
\end{tabular}

Nguồn: Kết quả xử lý từ dữ liệu điều tra

\subsection{Phân tích nhân tố khẳng định CFA}

Kết quả kiểm định CFA cho từng khái niệm trong thang đo cho thấy, tất cả chi-quare có giá trị P-value $>5 \%$; các giá trị $\mathrm{CMIN} / \mathrm{df} \leq 2, \mathrm{GFI}>0,8$, TLI và $\mathrm{CFI} \geq 0,9$. Như vậy $\mathrm{CFA}$ cho các khái niệm đều đạt tính đơn hướng, đảm bảo giá trị hội tụ, đảm bảo độ tin cậy và giá trị phân biệt.

Kết quả kiểm định mô hình tới hạn sau cùng cho thấy, mô hình có 309 bậc tự do, giá trị kiểm định chi-square $=575.542$ với $\mathrm{P}-$ Value $=0,000$, chi-square $/ \mathrm{df}=1,863$ đạt yêu cầu $<5$ (vì cỡ mẫu >200 - Kettinger và Lee, 1995) và các chỉ số chỉ ra mô hình phù hợp với dữ liệu thị trường $(\mathrm{GFI}=0,889, \mathrm{CFI}=0,951, \mathrm{TLI}=0,945$, và $\mathrm{RMSEA}=0,051)$. Ngoài ra, trọng số các biến quan sát đều đạt chuẩn cho phép $(>=0,5)$ và có ý nghĩa thống kê các giá trị $\mathrm{P}$ đều bằng 0,000 . 


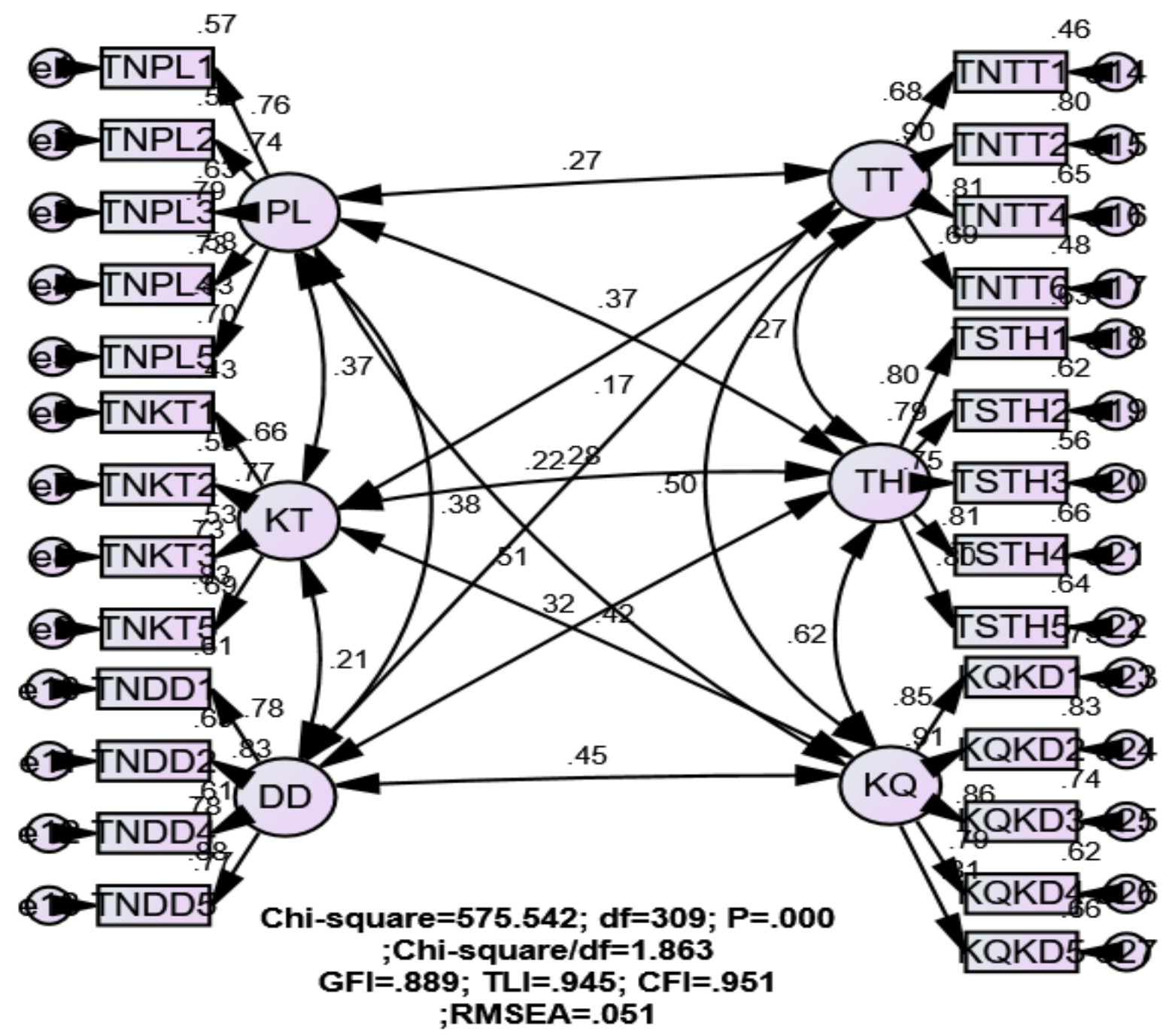

Hình 3. Kết quả CFA mô hình tới hạn

Như vậy CFA cho các khái niệm đều đạt tính đơn hướng, đảm bảo giá trị hội tụ, đảm bảo độ tin cậy và giá trị phân biệt. Mô hình nghiên cứu là phù hợp với dữ liệu thị trường.

\subsection{Phân tích mô hình cấu trúc tuyến tính SEM}

4.4.1. Kiểm định mô hình và các giả thuyết

Kết quả ước lượng (chuẩn hóa) mô hình nghiên cứu (Hình 3) cho thấy mô hình có 315 bậc tự do, giá trị kiểm định chi-square $=681.645$ với $\mathrm{P}$-Value $=0,000$, chi-square $/ \mathrm{df}=2.164<5$ và các chỉ số chỉ ra mô hình phù hợp với dữ liệu thị trường (GFI= 869, CFI = 933, TLI = 925, và $\mathrm{RMSEA}=0.059$ ) cho thấy mô hình phù hợp với dữ liệu nghiên cứu. 


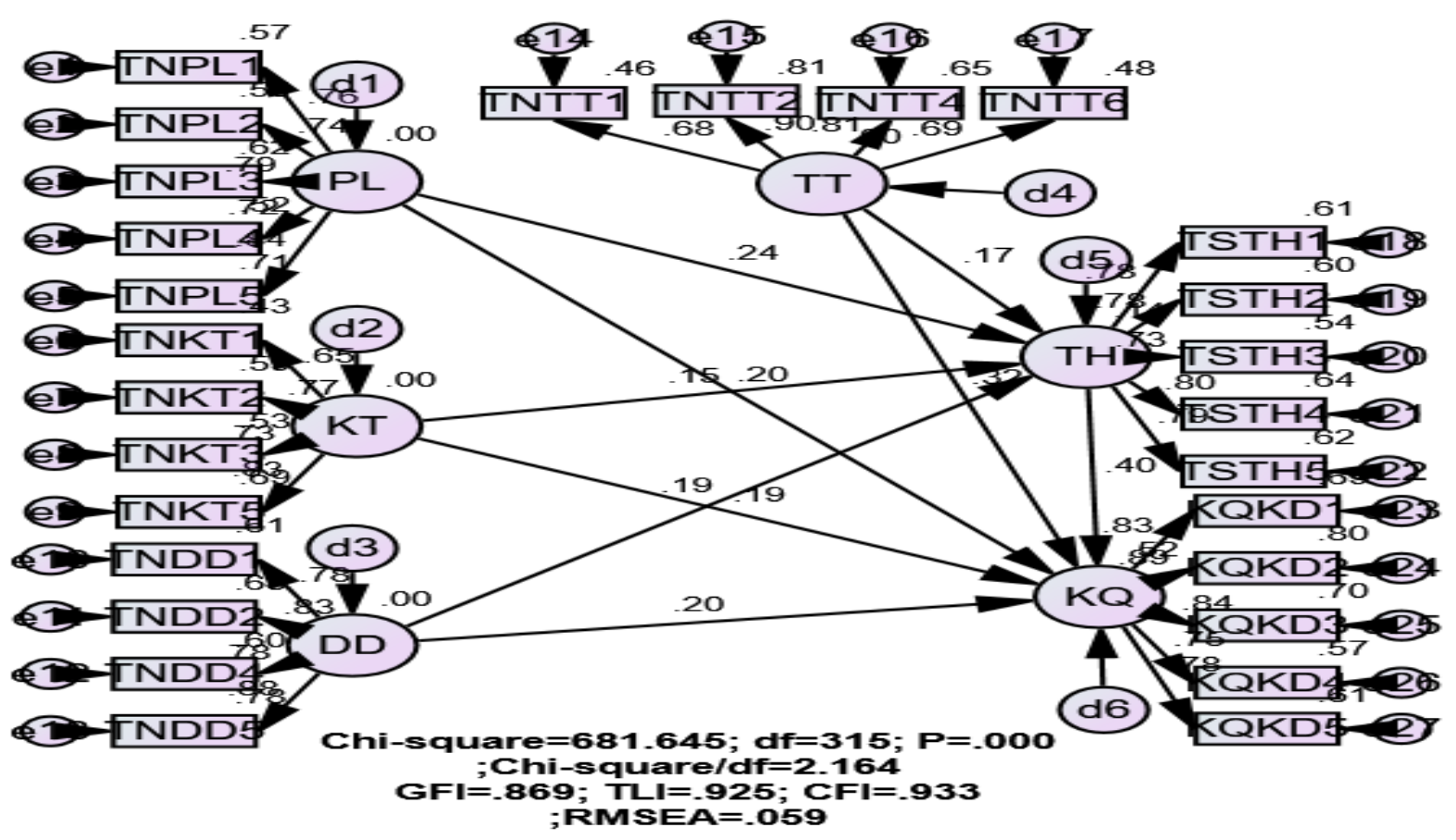

Hình 4. Kết quả mô hình SEM (chuẩn hóa)

\subsubsection{Kiểm định uớc luợng mô hình nghiên cưu bằng Bootstrap}

Kết quả ước lượng với $\mathrm{N}=500$ được tính trung bình kèm theo, cho thấy độ chệch có xuất hiện nhưng rất nhỏ. Vì vậy, có thể kết luận các ước lượng trong mô hình có thể tin cậy được.

\subsubsection{Kiểm định các giả thuyết nghiên cứu bằng mô hình cấu trúc tuyến tính SEM}

Kết quả ước lượng mô hình nghiên cứu trong mô hình cấu trúc tuyến tính SEM cho thấy, các mối quan hệ được giả thuyết trong mô hình nghiên cứu chính thức có giá trị thống kê vì có $\mathrm{p}$ có giá trị cao nhất là nhỏ hơn 0,05 , đạt ý nghĩa cần thiết (ở độ tin cậy $95 \%$ ). Nói cách khác, các giả thuyết trong mô hình nghiên cứu chính thức đều được chấp nhận.

\section{Bảng 4}

Hệ số hồi quy của mô hình nghiên cứu chính thức

\begin{tabular}{|c|c|c|c|c|c|c|c|c|}
\hline \multicolumn{2}{|c|}{ Quan hệ } & Hệ số & Hệ số chuẩn hóa & S.E. & C.R. & P & Kết luận \\
\hline TH & $<---$ & PL & 0.182 & 0.239 & 0.0480 & 15.869 & 0.000 & Phân biệt \\
\hline TH & $<---$ & TT & 0.176 & 0.169 & 0.0487 & 17.072 & 0.000 & Phân biệt \\
\hline TH & $<---$ & KT & 0.125 & 0.148 & 0.0488 & 17.444 & 0.000 & Phân biệt \\
\hline TH & $<---$ & DD & 0.124 & 0.190 & 0.0485 & 16.706 & 0.000 & Phân biệt \\
\hline KQ & $<---$ & TT & 0.433 & 0.319 & 0.0468 & 14.549 & 0.000 & Phân biệt \\
\hline KQ & $<---$ & PL & 0.201 & 0.202 & 0.0484 & 16.498 & 0.000 & Phân biệt \\
\hline KQ & $<---$ & KT & 0.208 & 0.189 & 0.0485 & 16.723 & 0.000 & Phân biệt \\
\hline KQ & $<---$ & DD & 0.17 & 0.200 & 0.0484 & 16.533 & 0.000 & Phân biệt \\
\hline KQ & $<---$ & TH & 0.527 & 0.404 & 0.0452 & 13.193 & 0.000 & Phân biệt \\
\hline
\end{tabular}

Nguồn: Kết quả phân tích dữ liệu của nhóm nghiên cứu 


\section{Thảo luận, hàm ý quản trị và hướng nghiên cứu trong tương lai}

\subsection{Thảo luận kết quả}

Kết quả mô hình SEM đã cho thấy các mối quan hệ cùng chiều giữa các yếu tố CSR đến tài sản thương hiệu và kết quả kinh doanh; và giữa tài sản thương hiệu và kết quả kinh doanh của doanh nghiệp.

Nghiên cứu này có điểm khác biệt so với các nghiên cứu trước; đó là đối tượng nghiên cứu là nhân viên các công ty du lịch tại Việt Nam, một quốc gia đang phát triển. Tuy nhiên, kết quả nghiên cứu có những nét tương đồng so với các nghiên cứu trước đây trên thế giới. Cảm nhận của nhân viên về các yếu tố cấu thành CSR tác động thuận chiều đến tài sản thương hiệu (tương đồng với nghiên cứu của Lai et al., 2010). Bên cạnh đó, cảm nhận của nhân viên về các yếu tố cấu thành CSR tác động thuận chiều đến kết quả kinh doanh; điều này tương đồng với các nghiên cứu (Chu \& Yang, 2009; Griffin \& Mahon, 1997; Peters \& Mullen, 2009; Verschoor, 1998; Wang et al., 2012). Ngoài ra, tài sản thương hiệu tác động thuận chiều đến kết quả kinh doanh của doanh nghiệp; điều này tương đồng với các nghiên cứu (Ayuso et al., 2006; Dangelico et al., 2013; Morgan et al., 2009; Vorhies et al., 2011; Weerawardena et al., 2006). Như vậy, mặc dù có sự khác biệt giữa đặc điểm của quốc gia và ngành kinh doanh nhưng có thể khẳng định rằng sự tác động của nhận thức về CSR của nhân viên đến tài sản thương hiệu và kết quả kinh doanh có điểm tương đồng. Doanh nghiệp cần nâng cao nhận thức của nhân viên về thực tiễn thực hiện trách nhiệm xã hội của mình; huy động nhân viên tự nguyện hợp tác và tuân thủ chiến lược CSR; qua đó góp phần phát triển tài sản thương hiệu và nâng cao kết quả kinh doanh của công ty.

\subsection{Hàm ý quản trị}

Dưới đây là một số hàm ý quản trị làm tăng tài sản thương hiệu và kết quả kinh doanh của các công ty du lịch dựa trên các yếu tố của CSR.

Về trách nhiệm pháp lý:

Các doanh nghiệp du lịch cần tuân thủ và thực hiện đúng các quy định của pháp luật trong kinh doanh. Đầu tiên cần quan tâm đến người lao động cả về vật chất lẫn tinh thần thông qua chế độ lương, thưởng, phúc lợi và bảo hiểm; tạo môi trường làm việc lành mạnh và vừa sức; có trách nhiệm phát triển đào tạo cho nhân viên, đảm bảo an toàn vệ sinh trong lao động và không lạm dụng lao động trẻ em; tôn trọng quyền bình đẳng nam, nữ dựa trên công bằng về năng lực của mỗi người trong tuyển dụng; không phân biệt đối xử về mặt sắc tộc, với người bị khuyết tật, với quá khứ của người lao động.

Hướng dẫn nhân viên tuân thủ Luật Du lịch, Luật Bảo vệ lợi ích người tiêu dùng; trong đó cần chú ý thực hiện đầy đủ các nghĩa vụ đã cam kết với khách du lịch; Áp dụng biện pháp bảo đảm an toàn tính mạng, sức khoẻ, tài sản của khách du lịch; tối thiểu hóa những tranh chấp dân sự phát sinh đối với du khách.

Về trách nhiệm đạo đức:

Các doanh nghiệp du lịch cần hướng dẫn nhân viên tuân thủ chuẩn mực đạo đức trong kinh doanh, đạo đức nghề nghiệp; cam kết và giữ chữ tín với các bên liên quan, nhất là đối tác 
và khách hàng. Ngoài ra, trách nhiệm đạo đức còn được thể hiện trong văn hóa giao tiếp trong nội bộ doanh nghiệp, giữa nhân viên với cấp trên và giữa nhân viên với nhau. Doanh nghiệp du lịch cần xây dựng tốt kênh truyền thông nội bộ để tiếp nhận phản hồi từ nhân viên; phát huy văn hóa phê bình trên nguyên tắc: "Phê bình khiển trách trong bầu không khí hòa hợp, không đối đầu, Win - Win". Tạo điều kiện phát triển nhân viên và đào tạo về du lịch bền vững; góp phần phát triển nguồn lao động du lịch có kỹ năng tại địa phương; phát triển các sản phẩm du lịch có trách nhiệm bền vững về kinh tế.

\section{Về trách nhiệm kinh tế:}

Hoạch định tầm nhìn, chiến lược phát triển dài hạn, trong đó chú trọng thực hiện du lịch có trách nhiệm. Cần liên tục nâng cao chất lượng sản phẩm dịch vụi nâng cao phong cách phục vụ như thông tin rõ ràng, công khai, trung thực số lượng, chất lượng, giá cả các dịch vụ, hàng hóa cung cấp cho khách du lịch, tránh trường hợp chèo kéo du khách, nâng giá sản phẩm dịch vụ. Không ngừng thúc đẩy năng suất làm việc của nhân viên. Tham gia chuỗi cung ứng bền vững bao gồm việc đảm bảo các nhà cung cấp sản phẩm và dịch vụ tuân theo các trách nhiệm với môi trường, xã hội và kinh tế; qua đó nâng cao nhận thức về tính bền vững trong nội bộ doanh nghiệp để tăng hiệu quả nhân viên và với các nhà cung cấp; doanh thu tăng qua các hành động giảm thiểu chi phí như việc giảm tiêu thụ năng lượng, nâng cao tính hiệu quả hoạt động bằng cách sử dụng hiệu quả các nguồn lực; ngoài ra doanh thu tăng do tăng số lượng du khách bởi nhu cầu về các sản phẩm du lịch có trách nhiệm đang tăng cao.

\section{Về trách nhiêm tù thiên:}

Đây là một hoạt động truyền thống, các doanh nghiệp tích cực tham gia các hoạt động đóng góp như ủng hộ các vùng bị thiên tai, ủng hộ phong trào xóa đói giảm nghèo, các hoạt động đền ơn đáp nghĩa với các đối tượng người có công, ủng hộ những nhóm người thiệt thòi như khuyết tật, nạn nhân chiến tranh và một số có đóng góp cho phát triển địa phương như các công trình giáo dục, y tế, vui chơi cho trẻ em nghèo,... Bên cạnh đó, các doanh nghiệp du lịch cần phải quan tâm hỗ trợ bảo vệ môi trường điểm đến.

\subsection{Hạn chế và hướng nghiên cúu tiếp theo}

\subsubsection{Hạn chế của nghiên cúu}

Kết quả nghiên cứu chỉ mang tính đặc thù cho các doanh nghiệp du lịch tại Thành phố Hồ Chí Minh, chưa mang tính đại diện cho các địa phương khác trong cả nước.

Nghiên cứu chưa đi sâu vào phân tích mối quan hệ giữa các doanh nghiệp trong chuỗi các doanh nghiệp du lịch của Thành phố để thấy được vai trò của mình trong hệ thống.

\subsubsection{Hương nghiên cúu tiếp theo}

Thứ nhất, mở rộng nghiên cứu cho các địa phương khác trong cả nước.

Thứ hai, nghiên cứu tiếp theo cần xét đến mối quan hệ giữa các doanh nghiệp du lịch này trong chuỗi doanh nghiệp du lịch của địa phương để thấy được vai trò của mình trong hệ thống. 
Thứ ba, nghiên cứu tiếp theo nên mở rộng nghiên cứu ở những lĩnh vực kinh doanh khác.

\section{Tài liệu tham khảo}

Aaker, D. A. (1991). Managing brand equity. New York, NY: The Free Press.

Abdolvand, M., \& Charsetad, P. (2013). Corporate social responsibility and brand equity in industrial marketing. International Journal of Academic Research in Business and Social Sciences, 3(9), 271-284.

Ackerberg, D. A. (2001). Empirically distinguishing informative and prestige effects of advertising. The RAND Journal of Economics, 32(2), 316-333.

Ayuso, S., Rodríguez, M. A., \& Ricart, J. E. (2006). Using stakeholder dialogue as a source for new ideas: A dynamic capability underlying sustainable innovation. Corporate Governance, 6(4), 475-490.

Backhaus, K., Steiner, M., \& Lügger, K. (2011). To invest, or not to invest, in brands? Drivers of brand relevance in B2B markets. Industrial Marketing Management, 40(7), 10821092.

Baumgarth, C., \& Binckebanck, L. (2011). Sales force impact on B-to-B brand equity: Conceptual framework and empirical test. The Journal of Product and Brand Management, 20(6), 487-498.

Baumgarth, C., \& Schmidt, M. (2010). How strong is the business-to-business brand in the workforce? An empirically-tested model of 'internal brand equity' in a business-tobusiness setting. Industrial Marketing Management, 39(8), 1250-1260.

Biedenbach, G., \& Marell, A. (2010). The impact of customer experience on brand equity in a business-to-business services setting. Journal of Brand Management, 17(6), 446-458.

Bowen, H. (1953). Social responsibility of the businessman. New York, NY: Harper and Row.

Brammer, S., \& Millington, A. (2008). Does it pay to be different? An analysis of the relationship between corporate social and financial performance. Strategic Management Journal, 29(12), 1325-1343.

Brickley, J., Smith, C., \& Zimmerman, J. (2002). Business ethics and organizational architecture. Journal of Banking \& Finance, 26(9), 1821-1835.

Brown, B. P., Bellenger, D. N., \& Johnston, W. J. (2007). The implications of business-tobusiness and consumer market differences for B2B branding strategy. Journal of Business Market Management, 1(3), 209-229.

Buzzell, R. D., \& Gale, B. T. (1987). The PIMS principles: Linking strategy to performance. New York, NY: The Free Press.

Carroll, A. B. (1979). A three-dimensional conceptual model of corporate social performance. Academy of Management Review, 4(4), 497-505. 
Carroll, A. B. (1991). The pyramid of corporate social responsibility: Toward the moral management of organizational stakeholders. Business Horizons, 34(4), 39-48.

Carroll, A. B., \& Shabana, K. M. (2010). The business case for corporate social responsibility: A review of concepts, research and practice. International Journal of Management Review, 12(1), 85-105.

Chaudhuri, A. (2002). How brand reputation affects the advertising-brand equity link. Journal of Advertising Research, 42(3), 33-43.

Chu, C. F., \& Yang, P. P. (2009). Empirical examination of relationship between corporate social responsibility and financial performance. Financial Forum, 7, 135-137.

Chu, S., \& Keh, H. T. (2006). Brand value creation: Analysis of the interbrand-business week brand value rankings. Marketing Letters, 17(4), 323-331.

Collier, J., \& Esteban, R. (2007). Corporate social responsibility and employee commitment. Business Ethics, 16(1), 19-33.

Cretu, A. E., \& Brodie, R. J. (2007). The influence of brand image and company reputation where manufacturers market to small firms: A customer value perspective. Industrial Marketing Management, 36(2), 230-240.

Cyert, R., \& March, J. G. (1992). A behavior theory of the firm (2nd ed.). Oxford, UK: Basil Blackwell.

Dangelico, R. M., Pontrandolfo, P., \& Pujari, D. (2013). Developing sustainable new products in the textile and upholstered furniture industries: Role of external integrative capabilities. Journal of Product Innovation Management, 30(4), 642-658.

Davis, D. F., Golicic, S. L., \& Marquardt, A. J. (2008). Branding a B2B service: Does a brand differentiate a logistics service provider? Industrial Marketing Management, 37(2), 218227.

Demetriou, A., Mouyi, A., \& Spanoudis, G. (2010). The development of mental processing. In W. F. Overton (Ed.), Biology, cognition and methods across the life-span: Handbook of life-span development (pp. 306-343). Hoboken, NJ: Wiley.

Dinh, N. D. (2016). TPP sẽ mang lại nhiều co hội cho du lịch Việt Nam [TPP will bring many opportunities for Vietnamese tourism]. Retrieved September 1, 2016, from http://www.trungtamwto.vn/tpp/tpp-se-mang-lai-nhieu-co-hoi-cho-du-lich-viet-nam.

Freeman, R. E. (1984). Strategic management: A stakeholder approach. Boston, MA: Pitman.

Griffin, J. J., \& Mahon, J. F. (1997). The corporate social performance and corporate financial performance debate: Twentyfive years of incomparable research. Business and Society, $36(1), 5-31$.

Grunenwald, J. P., \& Vernon, T. T. (1988). Pricing decision making for high-technology products and services. Journal of Business \& Industrial Marketing, 3(1), 61-70. 
Han, S. L., \& Sung, H. S. (2008). Industrial brand value and relationship performance in business markets - A general structural equation model. Industrial marketing management, 37(7), 807-818.

Heath, E. (2000). Key trends and challenges in destination marketing: The need for a new paradigm. In J. Ruddy \& S. Flanagan (Eds.), Tourism destination marketing: Gaining the competitive edge (pp. 123-135). Dublin, Ireland: Tourism Research Centre, Dublin Institute of Technology.

Hirose, Y. (2002). The report of the committee on brand valuation. Tokyo, Japan: Ministry of Economy, Trade and Industry, Government of Japan.

Ho, Y. K., Keh, H. T., \& Ong, J. (2005). The effects of R\&D and advertising on firm value: An examination of manufacturing and non-manufacturing firms. IEEE Transactions on Engineering Management, 52(1), 3-14.

Hoang, T. P. T., \& Huynh, L. H. (2015). Trách nhiệm xã hội của doanh nghiệp, niềm tin và sự gắn kết với tổ chức của nhân viên ngân hàng [Corporate social responsibility, trust and organizational cohesion of bank staff]. Tạp chí Phát triển Kinh tế, 26(8), 37-53.

Huang, C.-F., \& Ho, L. C. (2012). An empirical analysis of the influences of corporate social responsibility on organizational performance of Taiwan's construction industry: Using corporate image as a mediator. Construction Management and Economics, 30(4), 263275.

Hult, G. T. M., Hurley, R, F., \& Knight, G. A. (2004). Innovativeness: Its antecedents and impact on business performance. Industrial Marketing Management, 33(5), 429-438.

Hwang, S. N., \& Chang, T. Y. (2003). Using data envelopment analysis to measure hotel managerial efficiency change in Taiwan. Tourism Management, 24(4), 357-369.

Jensen, M. B., \& Klastrup, K. (2008). Towards a B2B customer-based brand equity model. Journal of Targeting, Measurement and Analysis for Marketing, 16(2), 122-128.

Jing, Z., Yanxin, J., Rizwan, S., \& Mingfei, D. (2015). Building industrial brand equity by leveraging firm capabilities and co-creating value with customers. Industrial Marketing Management, 51, 47-58.

Jones, R. (2005). Finding sources of brand value: Developing a stakeholder model of brand equity. Brand Management, 13(1), 10-32.

Keh, H. T., Nguyen, M. T. T., \& Hwei, P. N. (2007). The effect of entrepreneurial orientation and marketing information on the performance of SMEs. Journal of Business Venturing, 22(4), 592-611.

Keller, K. L. (1993). Conceptualizing, measuring, and managing customer-based brand equity. The Journal of Marketing, 57, 1-22.

Keller, K. L. (1998). Strategic brand management: Building, measuring, and managing brand equity. Upper Saddle River, NJ: Prentice Hall. 
Kim, J. H., \& Hyun, Y. J. (2011). A model to investigate the influence of marketing-mix efforts and corporate image on brand equity in the IT software sector. Industrial Marketing Management, 40(3), 424-438.

Kotler, P., \& Pfoertsch, W. (2007). Being known or being one of many: The need for brandmanagement for business-to-business companies. The Journal of Business and Industrial Marketing, 22(6), 357-362.

Laczniak, G., \& Murphy, P. (1991). Fostering ethical marketing decisions. Journal of Business Ethics, 10(4), 259-271.

Lai, C. S., Chiu, C. J., Yang, C. F., \& Pai, D. C. (2010). The effects of corporate social responsibility on brand performance: The mediating effect of industrial band equity and corporate reputation. Journal of Business Ethics, 95(3), 457-469.

Lussier, R. N. (2000). Management fundamentals. New York, NY: Thomson Learning Inc.

Maignan, I., \& Ferrell, O. C. (2004). Corporate social responsibility and marketing: An integrative framework. Journal of the Academy of Marketing Science, 32(1), 3-19.

Maignan, I., Ferrell, O. C., \& Ferrell, L. (2005). A stakeholder model for implementing social responsibility in marketing. European Journal of Marketing, 39(9/10), 956-977.

McGuire, J. (1963), Business and society. New York, NY: McGraw-Hill.

Mizik, N., \& Jacobson, R. (2003). Trading off between value creation and value appropriation: The financial implications of shifts in strategic emphasis. Journal of Marketing, 67(1), 63-76.

Morgan, N. A., Slotegraaf, R. J., \& Vorhies, D. W. (2009). Linking marketing capabilities with profit growth. International Journal of Research in Marketing, 26(4), 284-293.

Nguyen, D. T., \& Nguyen, T. M. T. (2009). Nghiên cúu khoa học trong quản trị kinh doanh [Scientific research in business administration]. Hanoi, Vietnam: Nhà xuất bản thống kê.

Nguyen, H. H. (2016). Anh hương của trách nhiệm xã hội doanh nghiệp tới lòng trung thành của khách hàng: Nghiên cứu trong ngành thức ăn chăn nuôi tại miền Bắc Việt Nam [Effect of corporate social responsibility on customer loyalty: Research in the feed industry in North Vietnam]. (Unpublished master's thesis). Trường Đại học Kinh tế Quốc dân, Hanoi, Vietnam.

Peters, R., \& Mullen, M. R. (2009). Some evidence of the cumulative effects of corporate social responsibility on financial performance. Journal of Global Business Issues, 3(1), 1-14.

Peterson, D. K. (2004). The relationship between perceptions of corporate citizenship and organizational commitment. Business \& Society, 43(3), 296-319. doi: $10.1177 / 0007650304268065$.

Pine, R., \& Phillips, P. (2005). Performance comparisons of hotels in China. International Journal of Hospitality Management, 24(1), 57-73. 
Preble, J. F., Reichel, A., \& Hoffman, R. C. (2000). Strategic alliances for competitive advantage: Evidence from Israel's hospitality industry. International Journal of Hospitality Management, 19(3), 327-341.

Preston, L. E., \& O'Bannon, D. P. (1997). The corporate social-financial performance relationship: A typology and analysis. Business and Society, 36(4), 419-429.

Roberts, J., \& Merrilees, B. (2007). Multiple roles of brands in business-to-business services. The Journal of Business and Industrial Marketing, 22(6), 410-417.

Rupp, D. E., Gananpathy, J., Aguilera, R. V., \& Williams, C. A. (2006). Employees' reactions to corporate social responsibility: An organizational justice framework. Journal of Organizational Behaviour, 27, 537-543.

Shaw, G. \& Williams, A. (1990). Tourism, economic development and the role of entrepreneurial activity. Progress in Tourism. Recreation and Hospitality Management, $2,67-81$.

Sims, R. L., \& Kroeck, K. G. (1994). The influence of ethics fit on employee satisfaction, commitment and turnover. Journal of Business Ethics, 10(4), 259-271.

Smith, W., \& Higgins, M. (2000). Cause related marketing: Ethics and the ecstatic, Business and Society, 39(3), 304-322.

Sweetin, V. H., Knowles, L. L., Summey, J. H., \& McQueen, K. S. (2013). Willingness-to punish the corporate brand for corporate social irresponsibility. Journal of Business Research, 66(10), 1822-1830.

Torres, A., Bijmolt, T. H., Tribo, J. A., \& Verhoef, P. (2012). Generating global brand equity through corporate social responsibility to key stakeholders. International Journal of Research in Marketing, 29(1), 13-24.

Van Riel, A. C. R., de Mortanges, C. P., \& Streukens, S. (2005). Marketing antecedents of industrial brand equity: An empirical investigation in specialty chemicals. Industrial Marketing Management, 34(8), 841-847.

Verschoor, C. C. (1998). A study of the link between a corporation's financial performance and its commitment of ethics. Journal of Business Ethics, 17(13), 1509-1516.

Villas-Boas, J. M. (2004). Consumer learning, brand loyalty, and competition. Marketing Science, 23(1), 134-145.

Vogel, D. (2004). Is there a market for virtue? A critical appraisal of corporate social responsibility. Paper presented at the Third Transatlantic Business Ethic Conference, Rutgers Business School Newark, NJ.

Vorhies, D. W., Orr, L. M., \& Bush, V. D. (2011). Improving customer-focused marketing capabilities and firm financial performance via marketing exploration and exploitation. Journal of the Academy of Marketing Science, 39(5), 736-756.

Wang, D. H.-M., Yu, T. H.-K., \& Liu, H. Q. (2013). Heterogeneous effect of high-tech industrial R\&D spending on economic growth. Journal of Business Research, 66(10), 1990-1993. 
Wang, Y. G., Hsu, W. L., \& Chang, K. (2012). The relationship between corporate social responsibility and firm performance: An application of quantile regression. Frontiers of Business Research in China, 6(2), 218-244.

Weerawardena, J., O'Cass, A., \& Julian, C. (2006). Does industry matter? Examining the role of industry structure and organizational learning in innovation and brand performance. Journal of Business Research, 59(1), 37-45.

Wu, F., \& Cavusgil, T. (2006). Organizational learning, commitment, and joint value creation in inter-firm relationships, Journal of Business Research, 59, 81-89.

You, C. S., Huang, C. C., Wang, H. B., Liu, K. N., Lin, C. H., \& Tseng, J. S. (2013). The relationship between corporate social responsibility, job satisfaction and organizational commitment. International Journal of Organizational Innovation, 5(4), 65-77.

Yu, T. H. K., Wang, D. H. M., \& Wu, K. L. (2015). Reexamining the red herring effect on healthcare expenditures. Journal of Business Research, 68(4), 783-787. 